\title{
Safety risk simulation of an airborne self separation concept of operation
}

\author{
Henk A.P. Blom ${ }^{1}$, Bart Klein Obbink ${ }^{2}$, G.J. (Bert) Bakker ${ }^{3}$ \\ National Aerospace Laboratory NLR, Amsterdam, The Netherlands
}

\begin{abstract}
[Abstract] This paper evaluates through Monte Carlo (MC) simulation an airborne self separation concept which has been developed for use in en-route traffic conditions such as encountered over the Mediterranean area. For three different encounter scenarios, probabilities for violating minimum separation and for near-mid-air and mid-air events are estimated by applying powerful novel MC simulation approaches in rare event estimation. This provides great new insight in the efficacy of airborne conflict resolution management. The paper shows several quantitative risk estimates and presents an interpretation of these results in terms of safety. It shows that airborne self separation can be very effective, but also has its limitations for dense traffic conditions when conflict resolution is done in a sequential and un-coordinated way.
\end{abstract}

\section{Introduction}

The aim of this paper is to present an initial safety risk assessment using Monte Carlo (MC) simulation of an airborne self-separation concept of operation under demanding traffic conditions. In Ref.1 such a study has been performed for self-separation equipped aircraft that were assumed to fly within a conventional fixed route structure. The current paper studies a true airborne self separation concept of operations, i.e. one without using fixed route structure. Earlier simulation studies of airborne self separation without route structure focused their analysis on safety in terms of conflict probability (e.g. Refs. 2, 3 and 4). These papers clearly demonstrated the kind of challenges these simulations have to deal with. In the current study, however, we go further and simulate up to the level of mid-air collisions. The specific airborne self separation application for which we conduct such simulations, has been developed for air traffic in the Mediterranean area (Ref. 5). For short we refer to this operational concept as AMFF (Autonomous Mediterranean Free Flight).

The capability to perform MC simulations up to collisions for airborne self separation without route structure, has been acquired through a sequence of theoretical studies and developments in the area of rare event estimation. The main results of these developments are:

- An approach towards the systematic specification of the AMFF operational concept in terms of a mathematically unambiguous model (Ref. 6), which supports the use of two complementary evaluation approaches: i) MC simulation of this model, and ii) stochastic analysis of this model in order to support rare event importance sampling.

- An extension of a powerful MC simulation importance sampling approach for estimating rare events (Ref.

7) and its application to mid-air collision risk assessment of airborne self separation (Refs. 8, 9, 10 and 11).

Rather than elaborating on these powerful novel approaches, the aim of this paper is to explain the MC simulation results obtained by applying these approaches towards demanding scenarios within the AMFF operational concept setting. In Ref. 12 we gave an initial presentation of the collision risk estimation results. The current paper further elaborates the results obtained:

- To systematically show what these MC simulation results mean for the conflict resolution phases that pass from medium term conflict through short term conflict to near mid-air.

- To explain the MC simulation results obtained for the model with results obtained for a gas model, with expectations of the concept design experts, and with an ED78a safety analysis (Ref. 13).

\footnotetext{
${ }^{1}$ blom@nlr.nl, NLR Air Transport Safety Institute, Fellow, IEEE.

2 bklein@nlr.nl, NLR Air Transport Safety Institute.

3 bakker@nlr.nl, NLR Air Transport Safety Institute.
} 
Preprint Proceedings $7^{\text {th }}$ AIAA-ATIO Conference, September 18-20, 2007, Belfast, Northern Ireland

- To develop valuable argumentation of what these simulation results mean for the AMFF operational concept considered and for airborne self separation in general.

The paper is organized as follows. Section II shortly presents the airborne self separation concept considered. Section III presents a high level view of the MC simulation model. Section IV presents MC simulation results for a two aircraft encounter scenario. Section V presents MC simulation results for an eight aircraft encounter scenario. Section VI presents MC simulation results for a dense random traffic scenario. Section VII compares AMFF with coordinated multi-aircraft conflict resolution. Section VIII draws conclusions.

\section{Airborne self separation concept considered}

For a complete description of the AMFF operational concept we refer to Ref. 5 and Ref. 14. In addition, Ref. 15 describes the background of the AMFF design philosophy. One important guideline in the development of the concept is the pilot's acceptability, including the comprehensibility of the conflict resolution maneuvers. This guideline, and the attempt to avoid vulnerabilities in the information exchange between aircraft, has led to the following AMFF characteristics:

a) a state-based conflict detection and resolution concept (as opposed to an intent-based concept),

b) implicit co-ordination of maneuvers between aircraft involved in a conflict (as opposed to explicit coordination in which information on the trajectories is exchanged),

c) sequential multiple conflict resolution (as opposed to solving a multiple conflict in a concurrent way).

d) straightforward pilot rules and procedures (as opposed to involving dedicated decision-making by artificially intelligent systems) and

e) a level of automation in which the pilot follows the automatically generated conflict resolution advices by steering the aircraft (as opposed to a direct coupling between the conflict resolution equipment and the aircraft guidance and control equipment).

Although the conflict detection and resolution approach developed for AMFF has its roots in the modified potential field approach in Ref. 16, there are significant differences. The main difference is that conflict resolution in AMFF is intentionally designed to solve multiple conflicts one by one rather than applying one joint potential field resolution. The resulting AMFF design can be summarized as follows:

- Aircraft are equipped with Automatic Dependent Surveillance-Broadcast (ADS-B), which periodically broadcasts own aircraft state information, and continuously receives state information messages broadcasted by aircraft that fly within broadcasting range $(\sim 100 \mathrm{NM})$.

- Aircraft are equipped with a system referred to as Predictive ASAS (P-ASAS), which indicates which maneuvers should be avoided to maintain a conflict-free trajectory. It for example verifies if an aircraft can safely return to its flight plan after executing a conflict resolution maneuver.

- Aircraft are equipped with ASAS (Airborne Separation Assurance System), including conflict detection and resolution based on linear extrapolation of the momentaneous states of the aircraft.

- The vertical separation minimum is $1000 \mathrm{ft}$ and the horizontal separation minimum is $5 \mathrm{NM}$. A conflict is detected by ASAS if these separation minima will be infringed within a look-ahead time of 6 minutes.

- The conflict resolution process consists of two phases. During the first phase (predicted conflict is 6 to 3 minutes ahead), priority rules determine for each crew whether their aircraft should make a resolution maneuver or not, and if yes, which one-by-one conflict should be resolved first. If this approach does not timely solve conflict(s), then during the second phase (predicted conflict is 3 minutes or less ahead), both crews should make a resolution maneuver.

- Two conflict resolution maneuver options are presented: one in vertical and one in horizontal direction. The presence of other aircraft is not taken into account. Crew decides which option to execute.

- All aircraft use the same resolution algorithm, and all crew apply the same procedures.

- ASAS-related and surveillance information is presented to the crew through a Cockpit Display of Traffic Information (CDTI). 


\section{MC Simulation Model}

For the initial safety risk assessment of AMFF, an extended, dynamic-stochastic model has been built using the compositional specification power of Petri Nets (Ref. 6). Subsequently, stochastic analysis and Monte Carlo simulations are developed to evaluate the model (Refs. 8, 9, 10 and 11).

Main elements taken into account in the Petri net model are:

- The actual, physical state of a number of aircraft in a certain volume in airborne self separation airspace,

- Communication, navigation and surveillance; both the specific means (ADS-B receivers and transmitters, Flight Management Systems, GNSS (Global Navigation Satellite System) receivers, etcetera) and information flows of individual aircraft, as well as global aspects as frequency saturation or corruption of GNSS.

- The ASAS system of each aircraft includes P-ASAS, conflict detection, conflict alerting and conflict resolution functionality,

- The crew of each aircraft, including their state and intent situation awareness, their memory, their cognitive mode and their task performance. The latter contains a blue-print of the way tasks are executed, for example prioritizing emergency actions over conflict resolution and navigation tasks.

- Safety critical factors, such as turbulence, engine failures, cabin decompression, ADS-B receiver failure, ASAS system corruption, etcetera.

Important elements that are not yet taken into the model are Airborne Collision Avoidance System (ACAS) and Airline Operational Centers (AOC's).

When running the MC simulations, it is possible to keep track of the dynamically developing states of all agents involved. In particular, it is considered interesting to track the physical states of the aircraft and to record safety related events as defined in Table 1 below. These safety related events are MTC (Medium Term Conflict), STC (Short Term Conflict), MSI (Minimum Separation Infringement), NMAC (Near Mid-Air Collision) and MAC (MidAir Collision). Their definition is in terms of the prediction time over which the current state is extrapolated, and a test on the remaining minimum horizontal and vertical distance between aircraft centres.

MC simulations have been performed for three AMFF scenarios. The first scenario considers a head-on encounter of two aircraft. The second scenario considers a simultaneous

Table 1. Safety related events definition

\begin{tabular}{|l|c|c|c|c|c|}
\hline Event & MTC & STC & MSI & NMAC & MAC \\
\hline $\begin{array}{l}\text { Prediction time } \\
\text { (minutes) }\end{array}$ & 8 & 2.5 & 0 & 0 & 0 \\
\hline $\begin{array}{l}\text { Horizontal } \\
\text { distance }(\mathrm{Nm})\end{array}$ & 4.5 & 4.5 & 4.5 & 1.25 & 0.054 \\
\hline $\begin{array}{l}\text { Vertical } \\
\text { distance (ft) }\end{array}$ & 900 & 900 & 900 & 500 & 131 \\
\hline
\end{tabular}

scenario considers dense random traffic. The main output of a set of MC simulations constitutes of point estimates of the probabilities of safety related events. Due to practical limitations, a bias and uncertainty analysis has not yet been performed, which means that the results should be interpreted in a relative way only.

\section{Simulation of two head-on aircraft}

In this scenario, two aircraft start at the same flight level, some $250 \mathrm{~km}$ away from each other, and fly on opposite direction flight plans head-on with a ground speed of approximately $240 \mathrm{~m} / \mathrm{s}$. For this encounter it can analytically be evaluated that without any control in terms of conflict detection and resolution, the probabilities of MTC, SCT, MSI, NMAC and MAC would be 1.0, 1.0, 1.0, 1.0 and 0.85 respectively. The ratios between the do nothing model (i.e. gas model) and those assessed in the MC simulations can be considered as a measure for the efficacy of the conflict detection and resolution as provided by the AMFF concept.

By conducting MC simulations for the two aircraft encounter scenario under AMFF, the following probabilities on safety related events are estimated (Table 2). 
Apparently, AMFF conflict detection and resolution are quite effective in avoiding STC (factor equals $1.0 / 4.5 \mathrm{E}-4 \approx 2200$ ) in case of a head on encounter between two aircraft. The ratio between STC and MSI probabilities is a complementary factor of about 180 (= 4.5E-4 / 2.5E-6). Together this means that the AMFF operational concept is very effective in preventing MSI for a head on encounter between two aircraft.

In order to assess the dependability of the results on GNSS, ADS-B and ASAS systems, the parameter values specifying the availability of those systems have also been improved a factor 100, and MC simulations have been executed again. Figure 1 shows both the baseline results and the results when the availability is a factor 100 higher.

Figure 1 clearly shows that for the two aircraft head-on encounter, a 100fold improvement in the availability/reliability of GNSS, ADS-B and ASAS systems leads to a 100 -fold improvement of the factor between STC and MSI probabilities, whereas the ratio between the probabilities of higher or lower levels remains unchanged. This indicates that the main cause for collision risk in this scenario lies in the availability of GNSS, ADS-B and ASAS systems. Moreover, this result suggests that for a two aircraft encounter the AMFF concept can reduce the probabilities for MSI, NMAC and MAC by increasing availability of critical systems.

The MC simulation results also show that the estimated STC, MSI, NMAC and MAC probabilities are such low that straightforward MC simulation falls short in evaluating airborne self separation applications if these are based on a number of 10,000 runs or less.

In Ref. 13, a safety assessment of two aircraft encounters for the AMFF design has been performed according to the ED78a methodology of RTCA/Eurocae. The MSI and MAC probabilities are estimated in a static way by using a fault tree. Because the geometry of the encounters considered are not the same, a direct quantitative comparison is not feasible. From a qualitative perspective however, the two results are quite well in line on the following aspects:

- Both predict effective resolution impact between MTC and STC, and between STC and MSI.

- Both predict that collision risk decreases linearly with increasing availability and reliability of GNSS, ADS-B and ASAS systems.

In comparison to ED78a, the MC simulation approach provides a much better evaluation means for the stochastic dynamic behaviour of aircraft while conflicts are evolving, and to investigate the effect of varying key parameter values, such as the encounter geometry. This adds significantly to the insight of the safety aspects of a two aircraft encounter. Ref. 13 also identified a clear need to assess multi-aircraft encounter situations on collision risk, but did not know a way how to do so using the ED78a methodology.

\section{Simulation of an eight-aircraft encounter}

In the second scenario eight aircraft that fly at the same flight level, and have initial flight plans that would make all eight aircraft to fly through the same point in space at the same moment in time (if there would be no control). 
Without such control, the probability of MTC, SCT, MSI, NMAC and MAC for an individual aircraft would all be equal to 1.0 .

The outcomes of the MC simulation of AMFF for the eight- aircraft encounter scenario are compared to the probabilities obtained for two aircraft in scenario 1. The results for these two scenarios are presented in Figure 2, with at left the two aircraft encounter, and at right the eight aircraft encounter.

Figure 2 shows that the overall efficacy in this encounter is reduced significantly compared to the two aircraft encounter, especially in the phase prior to STC. At the same time, by comparing the STC-MSI, the MSI-NMAC and the NMAC-MAC levels, it is concluded that the concept has apparently still resolving power after STC's have occurred.

A more detailed evaluation of simulation results (by tracing back what happened prior to a simulated MAC event) has shown that such a MAC is typically caused by the following effect. A crew starts to solve a multiple conflict sequentially by executing a certain manoeuvre that resolves a conflict with one other aircraft. This manoeuvre may have three possible outcomes, or any combination of these three outcomes:

- It solves the conflict aimed for;

- It solves other conflicts by coincidence;

- It induces new conflicts.

All together, the coincidental, uncoordinated way of working on resolutions may delay the implementation of a joint conflict resolution.

Through additional MC simulations it has been shown that the MAC probability for the

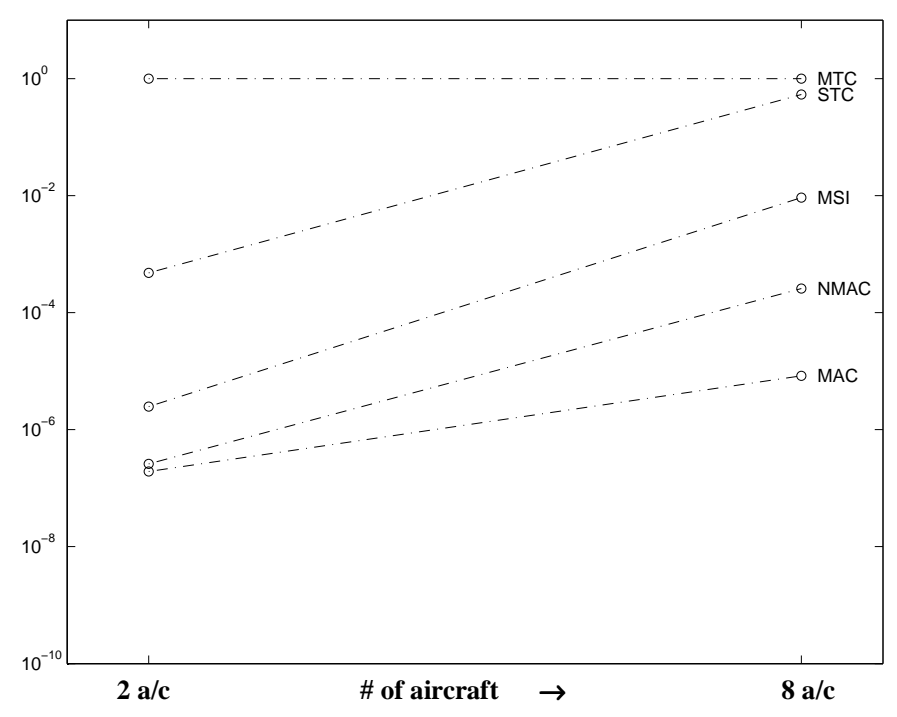

Figure 2. Two aircraft encounter vs. eight aircraft encounter eight encounter scenario is invariant with respect to the availability of GNSS, ADS-B and ASAS systems. This confirms that the main cause for collision risk in this multiple conflict scenario lies in the slowness in which multiple conflicts are resolved, which in turn can be due to the AMFF design approach to solve conflicts sequentially.

\section{Simulation of dense random traffic}

The third scenario artificially simulates aircraft flying randomly through a virtually unlimited Free Flight airspace by imposing periodic boundary conditions and random initial positions and velocities, after which traffic flies a sufficient period in time to obtain a steady state.

The gas model can be used to determine analytically the MTC, STC, MSI, NMAC and MAC probabilities in this scenario without any control in terms of conflict detection and resolution, depending on the aircraft density. This has provided positive verification of the results of MC simulations with GNSS, ADS-B and ASAS systems totally unavailable.

In a first series of MC simulations, the aircraft density of the third scenario is chosen three times the level of one of the busiest en-route sectors over Europe on $23^{\text {rd }}$ July 1999. The expected time to the conflict for an individual aircraft that is free of conflict is then only approximately five minutes. The efficacy of the AMFF concept can then be deduced by comparing the resulting estimates of the safety related events with the uncontrolled situation. Figure 3 presents the results for both these two situations.

Figure 3 shows that the efficacy of the AMFF concept is relatively low in this high traffic density; each of the probability values for the safety related events are higher than in the eight aircraft encounter scenario. The main cause for further detoriation appears to come from a less good ratio between the STC and MSI probabilities. Through further analysis of the simulation results it has been identified that the relative high risk is due to a small chance that multiple conflict situations occur and then such a cluster of conflicts tends to grow faster in size than the 
conflict resolution can handle. This in turn can again be due to the AMFF design approach to solve multiple conflicts in a sequential way.

Next MC simulations are performed for a 4 times lower traffic density, still about a factor 2.5 higher than the current mean en-route traffic density above Europe. Following the gas model (i.e. uncontrolled case) the MTC, STC, MSI, NMAC and MAC probabilities would all each reduce by a factor 4 . The simulation results of the AMFF controlled situation for both traffic densities are presented in fig. 4 .

Figure 4 shows that the MST, NMAC and MAC probabilities go down by a far larger factor than 4 (factor 70 for the MAC probability). This indicates that for AMFF the traffic density becomes critical at a certain point, in correspondence with expectations of the concept designers. It is furthermore noted that the resulting MAC probability in the scenario with medium traffic density is of the same order of magnitude (within a factor 4 ) as the one estimated for the two aircraft encounter scenario in Section IV. However, this is not true for the STC, the MSI and the NMAC probabilities. The estimated STC, MSI and NMAC probabilities for a dense random traffic scenario are much higher than these estimated for the two aircraft encounter. It shows that effective conflict resolution in this random traffic scenario is delayed to the late stages of conflict development, whereas these late stages hardly play a role in conflict resolution of a two aircraft head-on encounter. Another important difference is that the MAC probability in the two aircraft scenario can simply be reduced by improving the availability/reliability of GNSS, ADS-B and ASAS systems, whereas this option for improvement does not exist for the multi aircraft scenarios.

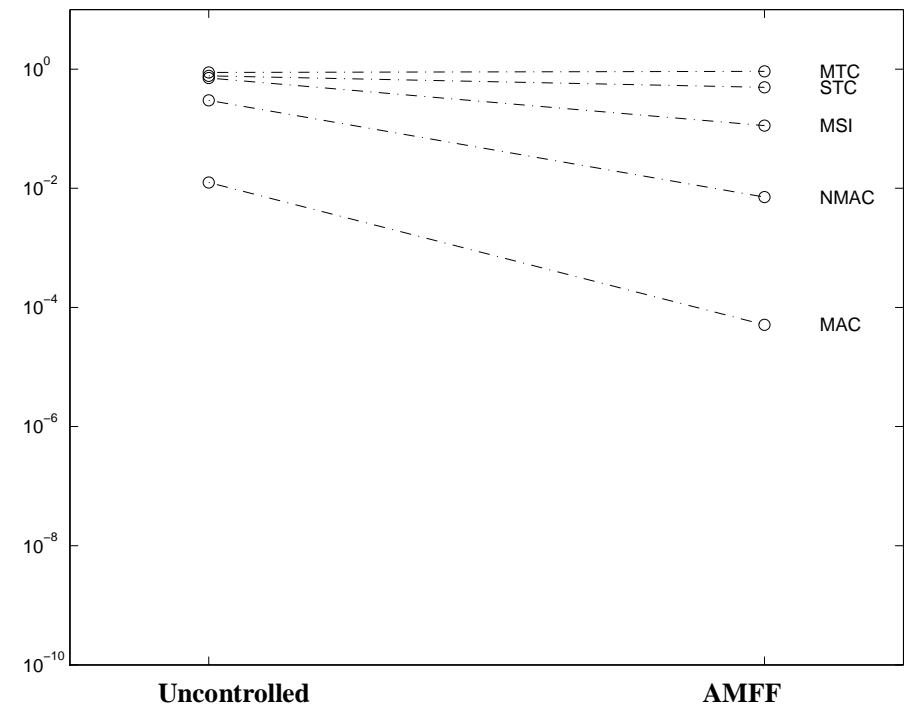

Figure 3. High density; uncontrolled vs. controlled

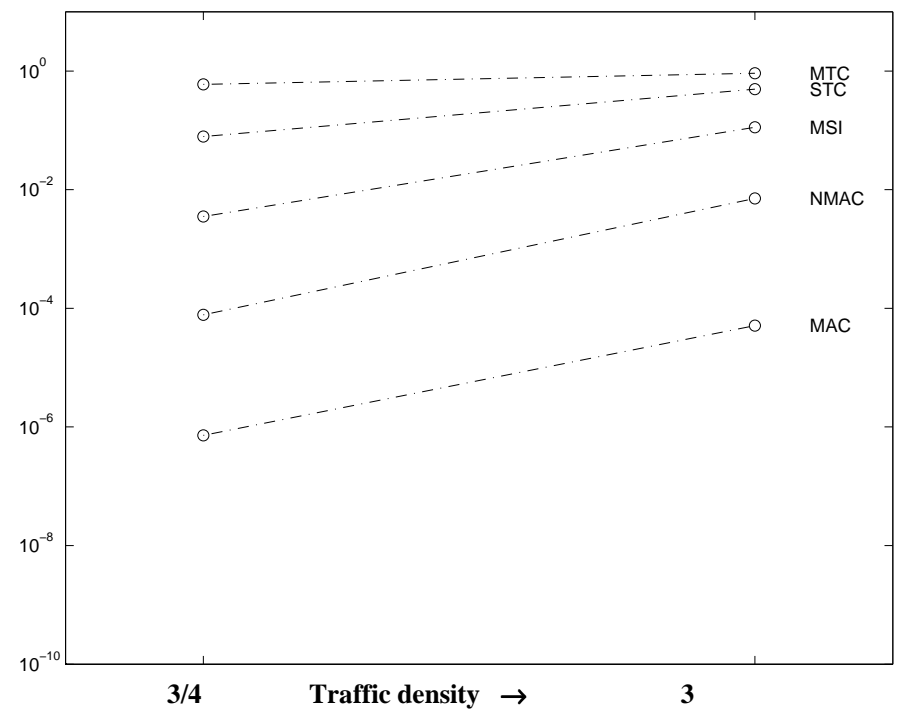

Figure 4. Variation of random traffic density (reference value is from a high density en-route sector over Europe in July 1999)

\section{Coordinated Conflict Resolution}

The MC simulation results for the two aircraft head on encounter shows that the self separation application under assessment can be very effective. Moreover, it turns out that in such a relative simple encounter, the collision risk depends directly on the availability and reliability of GNSS, ADS-B and ASAS systems. This shows that the AMFF 
concept can reduce the probabilities for MSI, NMAC and MAC in case of two aircraft encounters by increasing availability and reliability of GNSS, ADS-B and ASAS systems.

For the multi-aircraft scenarios in Sections V and VI, however, the results obtained show that the main cause for collision risk in such encounters stems from the slowness in which multiple conflicts are resolved. Hence the question is what the effect would be when the sequential un-coordinated conflict resolution would be replaced by a coordinated conflict resolution. In order to better understand this we take a better look at the trajectories that are generated under AMFF for this scenario. Figure 5a pictures an example of trajectories that are generated for the eight aircraft encounter scenario under the AMFF concept of operation. In order to provide an indication of distances, in the centre of the figure a $10 \mathrm{Nm}$ diameter circle is drawn. This shows that several aircraft make quite strange maneuvers under the AMFF concept. In order to make the picture of the maneuvers complete, fig.5b shows that some aircraft climb or descend. Because of random initial conditions and random disturbances, each MC simulated eight aircraft encounter yield patterns which are each time different from those in figs. 5a,b. The question one may pose is what would have happened when a coordinated conflict resolution approach had been used rather than the sequential and uncoordinated one of AMFF. For the eight aircraft encounter scenario, fig. 6 shows the trajectories that would be generated under the coordinated conflict resolution approach of Ref. 17. This clearly is much simpler and effective than the trajectories in fig.5a. Moreover, under the coordinated resolution approach none of the flights has to make a flight level change.

This comparison of AMFF generated resolution trajectories with optimal coordinated resolution trajectories clearly show that an important shortcoming of the AMFF design is its ineffectiveness to solve multiple conflicts in a well coordinated way, and this makes the AMFF design not suitable for dense traffic environments. Fortunately, the coordinated resolution example makes clear that this is not a fundamental limitation. It just was a limitation that 
AMFF designers have chosen for in order to accommodate pilot wishes. Hence, in order to safely accommodate very high en-route traffic demands through airborne self separation, the key question that should be addressed is how to take advantage of multi-aircraft coordinated conflict resolution approaches (e.g. Refs. 16, 17) such that pilot comprehensibility is well maintained.

\section{Concluding remarks}

The safety analysis of advanced operational concepts like airborne self separation has been recognized to be a problem that needs to be solved in order to enable a serious consideration of airborne self separation to be valid and feasible for application in busy en-route airspace. In order to improve this situation, the paper has evaluated several demanding airborne self separation scenarios on safety though estimating probabilities of rare events which range from Short Term Conflict through Minimum Separation Infringement to Near mid-air and Mid-air collision. This evaluation has become feasible due to a series of theoretical studies and developments in the area of MC simulation model development and MC speed up techniques in rare event estimation.

The airborne self separation concept that has been considered is referred to as AMFF. This AMFF concept has been very well developed for en-route airspace of low to moderate traffic demand, and it has been shown through real-time flight simulation studies that pilots experience it comfortable to fly under the AMFF concept. One of the features pilots appreciate of the AMFF concept is that ASAS has been designed such that it allows pilots to solve conflicts between aircraft in a sequential way rather than having to solve a multi-aircraft encounter problem in a coordinated way.

This paper has shown that an advanced MC simulation approach makes it possible to gain insight in safety related behaviour of such advanced concepts that has not be obtained with any of the traditional approaches towards safety analysis

Through these novel MC simulation results we learned understanding key safety related behaviour of this advanced design in a way that was not possible before. Sometimes the results are better than what the designers had expected and sometimes less good. In both cases the learning for the designers is very valuable. The less good findings do by no way mean that the concept airborne self separation is unsafe. The only thing it means is that the current results provide a nice opportunity for the designers of AMFF to start the development of innovative solutions for problems they did not observe before.

\section{References}

${ }^{1}$ M.H.C. Everdij, H.A.P. Blom, G.J. (Bert) Bakker. Modeling lateral spacing and separation for airborne separation assurance using Petri nets. Simulation (Transactions of the Society for Modelling and Simulation International), Vol. 83 (2007), forthcoming.

${ }^{2}$ J. Krozel, M. Peters, K. Bilimoria, C. Lee, J.S.B. Mitchell, System performance characteristics of centralized and decentralized air traffic separation strategies, Air Traffic Control Quarterly, Vol. 9 (2001), pp. 311-332.

${ }^{3}$ K.D. Bilimoria, H.Q. Lee, Properties of air traffic conflicts for free and structured routing, Proc. AIAA Guidance and Control Conference and Exhibition, 6-9 August 2001, Montreal, Canada. 
${ }^{4}$ M.R. Jardin, Analytical relationship between conflict counts and air-traffic density, AIAA J. Guidance, Control and Dynamics, Vol. 28 (2005), pp. 1150-1156.

${ }^{5}$ B. Gayraud, F. Nacchia, J. Barff, R.C.J. Ruigrok, MFF operational concept, requirements and procedures, Report MFF D220, October 2005, www.medff.it/public/index.asp

${ }^{6}$ M.H.C. Everdij, M.B. Klompstra, H.A.P. Blom, B. Klein Obbink, Compositional specification of a multi-agent system by stochastically and dynamically coloured Petri nets, Eds: H.A.P. Blom, J. Lygeros (eds.), Stochastic hybrid systems: Theory and safety critical applications, Springer, 2006, pp. 325-350.

${ }^{7}$ F. Cérou, P. Del Moral, F. Le Gland, P. Lezaud. Limit theorems for the multilevel splitting algorithms in the simulation of rare events. In Proceedings of Winter Simulation Conference, Orlando, USA, 2005.

${ }^{8}$ H.A.P. Blom, J. Krystul, G.J. Bakker, A particle systen for safety verification of free flight in air traffic, Proc. IEEE Conf. Decision and Control, San Diego, CA, 13-15 December 2006a.

${ }^{9}$ H.A.P. Blom, J. Krystul, G.J. Bakker, Estimating rare event probabilities in large scale stochastic hybrid systems by sequential Monte Carlo simulation, Proc. Int. Workshop on Rare Event SIMulation (RESIM), October 9-10, Bamberg, Germany, $2006 \mathrm{~b}$.

${ }^{10}$ H.A.P. Blom, J. Krystul, G.J. Bakker, M.B. Klompstra, B. Klein Obbink, Free flight collision risk estimation by sequential Monte Carlo simulation, Eds: C.G. Cassandras and J. Lygeros, Stochastic hybrid systems; recent developments and research trends, Taylor \& Francis/CRC Press, 2007, pp. 249-281.

${ }^{11}$ H.A.P. Blom, G.J. Bakker, J. Krystul, Reachability analysis for large scale stochastic hybrid systems, Forthcoming Proc. IEEE Conference on Decision and Control, December 12-14, 2007, New Orleans, USA.

${ }^{12}$ H.A.P. Blom, G.J. Bakker, B. Klein Obbink, M.B. Klompstra, Free flight safety risk modeling and simulation, Proc. Int. Conf. on Research in Air Transport (ICRAT), Belgrade, June 26-28, 2006c. 2005.

${ }^{13}$ MFF, MFF Final safety case, Report MFF D734, ed. 1.0. Available at http://www.medff.it/public/index.asp, November

${ }^{14}$ R.C.J. Ruigrok, N. de Gelder, H.J. Abma, B. Klein Obbink, J.J. Scholte, Pilot perspective of ASAS self separation in challenging environments, Proc. 6th USA/Europe ATM R\&D Seminar, Baltimore, USA, 27-30 June 2005.

${ }^{15}$ F. Maracich, Flying free flight: pilot perspective and system integration requirements, Proc. 24th DASC, Washington, 2005.

${ }^{16}$ J. Hoekstra. Designing for Safety, the Free Flight Air Traffic Management concept, PhD Thesis, Delft University of Technology, November 2001.

${ }^{17}$ I. Hwang, J. Kim, C. Tomlin, Protocol-based conflict resolution for air traffic control, Air Traffic Control Quarterly, Vol. 15, (2007), pp.1-34. 